\title{
Age of onset in Huntington's disease: Lack of parental age effect
}

\author{
WYLIE BURKE \\ From the Department of Pediatrics, University of Washington, School of Medicine, Seattle, Washington 98195, USA
}

Summary. In a recent publication Brackenridge and Teltscher (1975) concluded that the age of onset of Huntington's disease was in part a function of the age of the transmitting parent at the time of birth of a subsequently affected child. Their analysis suggested that the younger the parent was at the time of birth of the subsequently affected child, the later in life symptoms of disease would appear in the child. The data of Brackenridge and Teltscher have been statistically reevaluated here, and this analysis fails to support their conclusion. Consequently it would be irresponsible to counsel persons at risk for Huntington's disease to plan families early in life.

In a recent article published in this journal, Brackenridge and Teltscher (1975) claim that the age of onset of the autosomal dominant disorder, Huntington's disease, is in part a function of the age of the transmitting parent at the time of birth of a subsequently affected child. They analysed a group of kindreds in which Huntington's disease had segregated and concluded that the age of onset of the disease in affected children showed a positive linear regression with the parameter $P_{o}-P_{c}$, where $P_{0}$ is the age of onset of disease in the transmitting parent and $P_{c}$ is the age of the transmitting parent at the time of birth of the subsequently affected child. On the basis of their analysis, they advise that 'an important conclusion for genetic counselling is the desirability of parents at risk who intend to have children to plan their families early in life so that the illness will tend to appear in late adulthood in their affected children'.

The data used by Brackenridge and Teltscher were statistically re-evaluated here, and the parental age effect they described could not be substantiated. As a result it is important that individuals at risk for Huntington's disease should not be counselled to have children early in life. The negative effect of such counselling is further discussed below.

\section{Subjects and methods}

The data used were those of Brackenridge and Teltscher (1975), described in the Appendix to their

Received 19 September 1975. paper. For each progeny affected with Huntington's disease, the following parameters were determined from the data provided: $P_{0}$ (age of parent at onset of Huntington's disease), $P_{c}$ (age of parent at time of birth of subsequently affected child); $P_{o}-P_{c}$; and $C_{o}$ (age of child at onset of Huntington's disease). Parameter notation is the same as that used by Brackenridge and Teltscher There are well-recognized difficulties in fixing the age? of onset of clinical signs in this disease. In this study the age of onset as reported by Brackenridge and Teltscher has been used, without attempting to evaluate its accuracy.

Linear regressions were evaluated by calculating the coefficient of correlation, $r$, according to the usual product moment formula:

$$
r=\frac{\Sigma x y-\frac{\Sigma x \Sigma y}{n}}{\sqrt{\left(\Sigma x^{2}-\frac{(\Sigma x)^{2}}{n}\right)\left(\Sigma y^{2}-\frac{(\Sigma y)^{2}}{n}\right)}}
$$

The slope for each linear regression, $\mathrm{m}$, was also calculated, using the usual formula:

$$
\mathrm{m}=\frac{\Sigma \mathrm{xy}-\frac{\Sigma \mathrm{x} \Sigma \mathrm{y}}{\mathrm{n}}}{\Sigma \mathrm{x}^{2}-\frac{(\Sigma \mathrm{x})^{2}}{\mathrm{n}}}
$$

A multiple regression analysis of the data was also performed using the biomedical computer programme BMDO3R (Dixon, 1971) at the University of Washington Computer Center. For this analysis, $P_{0}$ was considered as the first independent variable and $P_{c}$ as the second independent variable. 
Further statistical manipulations are described in detail under 'Results'.

\section{Results}

A statistically significant correlation between age at onset of Huntington's disease in the transmitting parent and that in the affected child has previously been shown; for example, Brackenridge (1972) reported a correlation coefficient of 0.556 for these two parameters in subjects affected with Huntington's disease ascertained from published papers. Though statistically significant, this correlation is not large enough to be applied in predicting the age of onset for individuals at risk to develop Huntington's disease. It seems likely that other, possibly environmental, factors contribute to the variation in age of onset seen within a particular kindred. In their recent work, using a different sample of reported kindreds, Brackenridge and Teltscher (1975) claim that the age of the transmitting parent at the time of birth of a subsequently affected child is an additional measure which influences the age of onset of the disease. They conclude that the younger the transmitting parent is at the time of the child's birth, the later in life symptoms of disease will appear in the child. Their claim rests on their demonstration of a significant linear regression between 'mean onset age of offspring' and 'year of parental onset less year of birth of offspring;' (i.e., mean $C_{o}$ vs $P_{o}-P_{c}$, as defined above).

An important control in this analysis is the determination of the linear regression between age of onset in offspring and age of onset in parent (i.e., $C_{o}$ vs $P_{0}$ ) for the data used. As can be seen in Fig. 1, there is a significant linear regression of $C_{0}$ on $P_{o}$, with a coefficient of correlation, $r$, of 0.69 . This correlation is higher than the correlation of 0.556 previously reported by Brackenridge (1972) for a larger set of data $(N=250)$. In the present case the data were limited to those cases where the parameter $P_{c}$ was known (N=73, Brackenridge and Teltscher, 1975). Whether the difference in $r$ values is statistically significant is difficult to determine.* It is possible, however, that the data used by Brackenridge and Teltscher (1975) are not representative of Huntington's kindreds described in other reports.

These data thus show a significant correlation between the age of onset of Huntington's disease in the parent and the age of onset in the child. When the

\footnotetext{
* In each set of data affected individuals are grouped in kindreds and thus are not independent data points. To evaluate the degree of error in each calculation of $r$, one has to make an estimate of the degrees of freedom based on number of kindreds as well as number of affected individuals. The number of kindreds is not indicated for the larger set of data (Brackenridge, 1972).
}

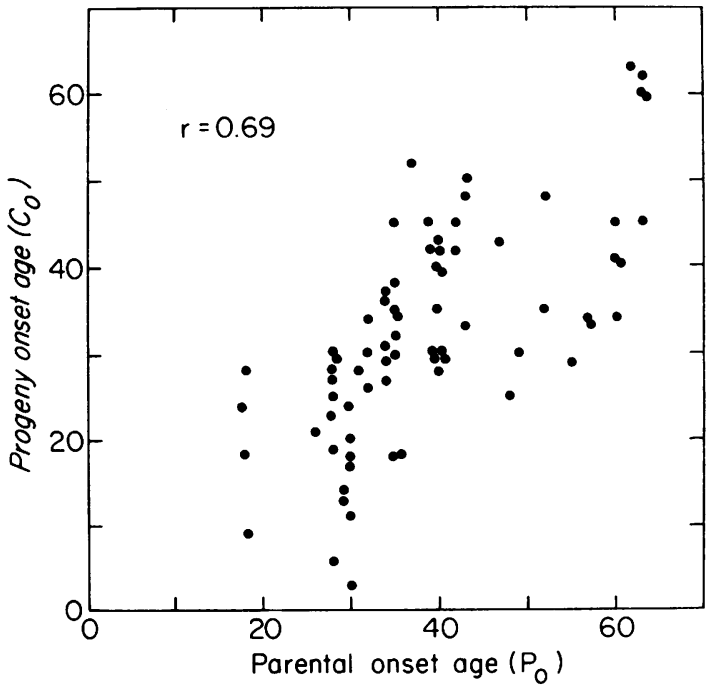

FIG. 1. The regression between age of onset in progeny $\left(\mathrm{C}_{0}\right)$ and age of onset in transmitting parent $\left(P_{0}\right)$. The coefficient of correlation, $r$, is 0.69 and the slope, $\mathrm{m}$, is 0.73 .

regression of $\mathrm{C}_{o}$ on the combined parameter $\mathbf{P}_{o}-\mathbf{P}_{c}$ (parental onset age less age of parent at birth of subsequently affected child) is plotted, there is no increase in correlation (Fig. 2). The values of the coefficients of correlation are indistinguishable,

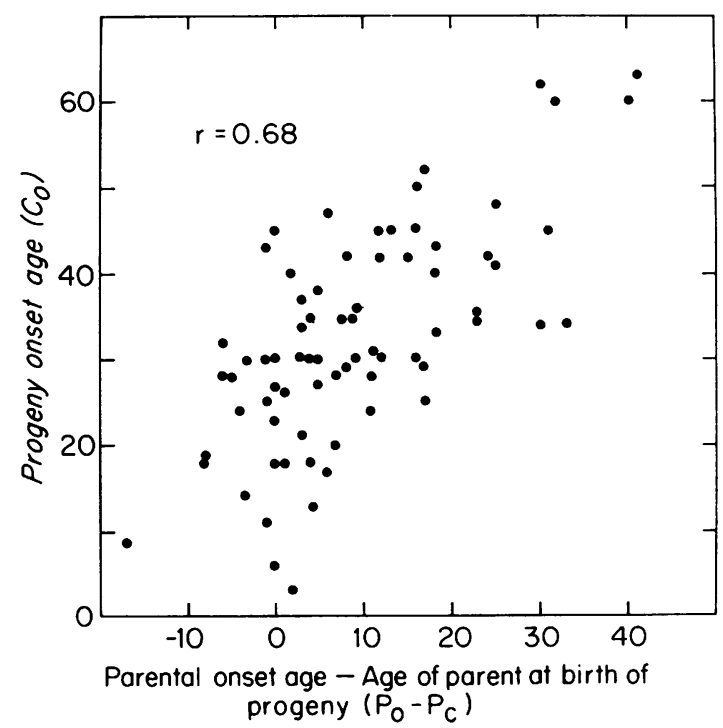

FIG. 2. The regression between age of onset in progeny $\left(\mathrm{C}_{0}\right)$ and the parameter $P_{0}-P_{c}$ (age on onset in transmitting parent-age of transmitting parent at time of birth of subsequently affected child). The coefficient of correlation, $\mathrm{r}$, is 0.68 and the slope, $\mathrm{m}$, is 0.73 . 
0.68 vs. 0.69 , and the slopes are identical, both 0.73 . This result shows that the ability to predict probable age of onset, for individuals at risk to develop Huntington's disease, is in no way increased by using the parameter $P_{o}-P_{c}$ instead of $P_{o}$. For purposes of genetic counselling, the age of the transmitting parent at the time of birth of a child has no effect on the age at which the child will show disease, if he carries the allele for Huntington's disease.

It should be noted that the plot shown in Fig. 2 differs significantly from the plot shown in Fig. 1 of Brackenridge and Teltscher's paper (1975), where 'mean onset-age of offspring' is plotted against 'year of parental onset less year of birth of offspring'. Though both figures present the degree of correlation between the parameter $\mathrm{C}_{o}$ and the parameter $P_{o}-P_{c}$, the Brackenridge and Teltscher plot has used mean values for $\mathrm{C}_{0}$, rather than incorporating each value of $\mathrm{C}_{o}$ as a single datum point, as is done here in Fig. 2. The use of mean values of $C_{0}$ results in an artificial increase in correlation between the two parameters. This is because averaging removes the variance contributed by each series of $\mathrm{C}_{0}$ values corresponding to a given value of $P_{o}-P_{c}$. With each reduction in variance, the correlation is correspondingly, and incorrectly, increased.

The relation between $C_{o}$ and the two variables $P_{o}$ and $P_{c}$ was further studied by performing a computerized multiple regression analysis (see Subjects and Methods). This analysis permits a measure of the degree to which each independent variable contributes to a regression between a dependent variable and two or more independent variables. When this analysis was performed using $P_{0}$ as the first independent variable and $P_{c}$ as the second independent variable, $P_{o}$ was shown to account for $45 \%$ of the variance in the regression between $\mathrm{C}_{0}$ and the two variables $P_{o}$ and $P_{c}$, while $P_{c}$ accounted for less than $3 \%$ of the variance. Thus, even with a more sophisticated analysis, the addition of the parameter $P_{c}$ cannot be shown to add significantly to the correlation observed between $\mathrm{C}_{0}$ and $\mathrm{P}_{0}$.

The conclusions of Brackenridge and Teltscher can be tested further by examining a prediction which follows from their results. If age of onset in affected progeny varies inversely with age of transmitting parent at the time of their birth, it follows that within any given kindred younger affected progeny should show an earlier age of onset than their older affected sibs.

One way of testing this prediction is to examine the relation between $C_{o}$ and $P_{o}-P_{c}$ within kindreds containing two or more affected children. Among the data used by Brackenridge and Teltscher, there

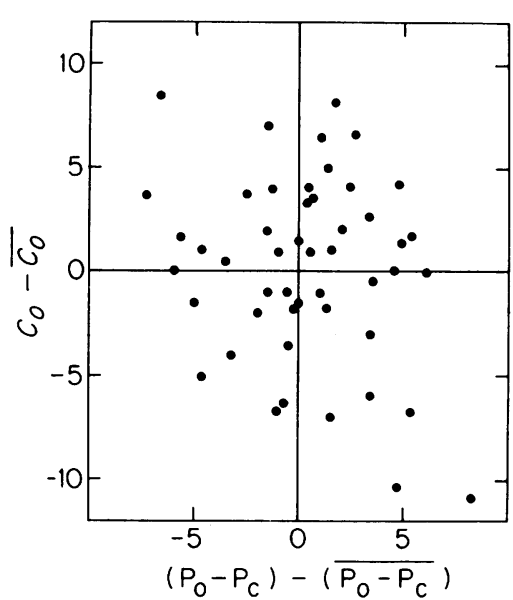

FIG. 3. The regression between $\mathbf{C}_{0}-\overline{\mathbf{C}_{0}}$ and $\left(\mathbf{P}_{0}-\mathbf{P}_{\mathrm{c}}\right)-\overline{\left(\mathbf{P}_{0}-\mathbf{P}_{c}\right)}$ for all kindreds containing two or more affected children. The coefficient of correlation, $\mathrm{r}$, is $-\mathbf{0 . 2 2}$ and the slope, $\mathrm{m}$, is -0.27 .

were 17 of these kindreds, with a total of 49 progeny. In some of these kindreds $(\mathrm{N}=5)$, there was a positive correlation between $\mathrm{C}_{o}$ and $\mathrm{P}_{\mathrm{o}}-\mathrm{P}_{\mathrm{c}}$, in some kindreds $(\mathrm{N}=5)$ a negative correlation, and in some kindreds $(\mathrm{N}=7)$ no apparent correlation. For a statistical evaluation of the data, the following method was used: within each kindred, $C_{0}$ and $\vec{\theta}$ $\mathrm{P}_{\mathrm{o}}-\mathrm{P}_{\mathrm{c}}$ were determined for each affected child, an $\overline{\mathrm{C}_{\mathrm{o}}}$ and $\overline{\mathrm{P}_{\mathrm{o}}-\mathrm{P}_{\mathrm{c}}}$ (mean values) were calculated for a data from the kindred. Then, for each affecte重 child, $\mathrm{C}_{\mathrm{o}}-\overline{\mathrm{C}_{\mathrm{o}}}$ and $\left(\mathrm{P}_{\mathrm{o}}-\mathbf{P}_{\mathrm{c}}\right)-\overline{\left(\mathrm{P}_{\mathrm{o}}-\mathrm{P}_{\mathrm{c}}\right)}$ were calculated. In other words, each of the $C_{o}$ and $P_{o}-P_{c}$ values was normalized by subtracting the mean $C_{o}$ or $P_{o}-P_{c}$ value for the kindred from it. By expressing each point as a deviation from the mean, it is possible to consider data from all kindreds within the same set of co-ordinates. This normalization process permits data from different kindreds to be compared directly. As shown in Fig. 3, a plot of the normalized data shows little, if any, discernible relation between the two measures. Calculation of the coefficient of correlation, $r$, for these data, gives a value of -0.22 . This correlation is small and of doubtful significance statistically,* but most im- 음 portant it is a negative correlation, where Brackenridge and Teltscher would predict a positive correlation. An examination of the relation between $\mathrm{C}_{\mathrm{o}} \mathrm{N}$ and $P_{o}-P_{c}$ in kindreds containing 2 or more of

* If one assumes $\mathrm{N}-2$ degrees of freedom for these data $(\mathrm{N}=$ num* If one assumes $\mathrm{N}-2$ degrees of freedom for these data $(N=n u m-$
ber of data points $=49)$ a t-test assigns a probability between 0.10 and $N$ 0.05 to this correlation. However, a strict evaluation of the data $\sigma$ suggests that there are fewer than $\mathrm{N}-2$ degrees of freedom, since data points within a given kindred are not independent of one another. 0 Assuming the loss of a degree of freedom for each kindred, the number of degrees of freedom for these data drops from 47 to 31 , and a $\mathbb{D}$ t-test fails to assign significant probability to the correlation. 
affected children thus provides direct evidence against the conclusions of Brackenridge and Teltscher.

\section{Discussion}

Finding new methods which help in predicting the expected age of onset for individuals at risk to develop Huntington's disease would be of great use in genetic counselling. Determining the factors which affect age of onset in this disease also would be of interest for an understanding of the pathophysiology of the disease. The incorrect identification of such factors, however, represents a roadblock to both effective genetic counselling and further understanding of the disease. For these reasons, it is important to correct the misunderstanding conveyed by Brackenridge and Teltscher in their recent publication. A re-evaluation of their data fails to support their conclusion that the age of onset of Huntington's disease is a function of the value $P_{o}-P_{c}$ (parental onset age less age of parent at birth of subsequently affected child). There is no basis for their contention that, 'a promising approach to select for onset in late adulthood' would be to encourage individuals at risk to have families early in life. On the contrary, such advice offers only a false hope for alleviating the burden of the disease in affecting offspring. This kind of counselling would be likely to have the negative effect of increasing the number of individuals with Huntington's disease. Not only would individuals at risk be encouraged to start families at an earlier age than they might otherwise have done, they would also do so in the belief that any children of theirs affected with Huntington's disease would have an onset of symptoms late in life. Such a belief might cause these individuals to be more willing to have children than they would otherwise have been.

The author wishes to thank $\mathrm{Dr}$ Joe Felsenstein for advice on the statistical analyses performed here and for comments on the manuscript, Dr Jurg Ott for help with the BMD computer programme, and Dr Gilbert Omenn for generous encouragement and critical reading of the manuscript.

This work was supported in part from grants HD04665 and HD-02274 from the National Institutes of Health.

\section{REFERENCES}

Brackenridge, C. J. (1972). The relation of some features of Huntington's disease to the age of onset of symptoms in parents. Clinical Genetics, 3, 477-482.

Brackenridge, C. J. and Teltscher, B. (1975). Estimation of the age of onset of Huntington's disease from factors associated with the affected parent. Fournal of Medical Genetics, 12, 64-69.

Dixon, W. J. (1971). (Ed.) BMD Biomedical Computer Programs, pp. 258-269. University of California, Berkeley. 